\title{
Improved Linewidth Tolerant Carrier Phase Recovery Based on Polar MAP Metric Estimate
}

\author{
Martí Sales*, Md. Saifuddin Faruk, and Seb J. Savory \\ Electrical Engineering Division, Engineering Department, University of Cambridge, U.K. \\ *ms2345@cam.ac.uk
}

\begin{abstract}
A new metric that analytically approximates the maximum a posteriori $(M A P)$ solution is presented. Used with a decision-directed carrier phase estimation algorithm, the linewidth tolerance exceeds the limits achieved when using the conventional Euclidean distance.

OCIS codes: (060.1660) Coherent communications; (060.2330) Fiber optics communications; (060.4080) Modulation
\end{abstract}

\section{Introduction}

Digital coherent receivers have become the preferred solution for current and next generation optical systems, including access networks [1], due to its ability to map the optical field onto the electrical domain and perform impairment compensation by means of digital signal processing techniques. A key algorithm in the DSP chain is the carrier phase estimation (CPE) which aims to overcome the transmitter and receiver lasers' phase noise.

There are two types of CPE algorithms: decision directed (DD) and non-decision directed (NDD). The most popular NDD algorithm is the Viterbi \& Viterbi algorithm [2] which uses the power-law to obtain the phase estimation and has become widely used with $M$-PSK constellations. For higher order QAM constellations there are two principal decision directed algorithms which are relevant to the scope of this paper: the maximum a posteriori (MAP) [3] and the blind phase search (BPS) [4] algorithms. In one hand, the MAP estimation is optimum in terms that it takes into account the parameters for both ASE and phase noise sources but solving it requires a computationally expensive iterative joint minimization process. In the other hand, BPS provides a hardware-efficient implementation and is considered the de facto benchmarking algorithm for CPE comparison with higher order quadrature amplitude modulation (QAM) systems. However, the decision mechanism in BPS relies on Euclidean distance, which lacks of rotational sensitivity.

In this paper, we propose a polar-based metric that finds the MAP estimate between two points in the plane in a closed-form solution. It will be shown that replacing the Euclidean distance with the proposed one in the BPS algorithm, the achievable linewidth tolerance limits can be improved significantly.

\section{Background}

The performance of CPE algorithms is usually evaluated across the figure combined linewidth-symbol period product $\left(\Delta v T_{s}\right)$ and assuming that the signal arriving to the block input has been perfectly equalized, and the frequency offset successfully removed. Thus, the incoming samples $y(k)$ can be expressed as follows:

$$
y(k)=x(k) e^{j \varphi(k)}+n(k)
$$

where $x(k)$ are the data symbols, $n(k)$ is the amplified spontaneous emission (ASE) noise described by a Gaussian process with variance $\sigma_{n}^{2}$ and $\varphi(k)$ is the combined transmitter and receiver phase noise which is modeled as a Wiener process with variance $\sigma_{p}^{2}=2 \pi \Delta v T_{s}$.

The MAP estimation obtains the maximum sequence probability by minimizing the log-probability function [3]:

$$
\log (P)=\sum_{k} \frac{|y(k)-x(k) \exp (j \varphi(k))|^{2}}{2 \sigma_{n}{ }^{2}}+\frac{(\varphi(k)-\varphi(k-1))^{2}}{2 \sigma_{p}{ }^{2}} .
$$

\section{Proposed metric definition: a polar based MAP approximation (pMAP)}

Since Eq. (2) does not yield a closed form solution, the proposed method consists of calculating the squared Euclidean distance following the cosine rule by which the squared of the distance $D$ shown in Fig. 1(a) can be calculated as

$$
D^{2}=|x|^{2}+|y|^{2}-2|x| \cdot|y| \cos (\alpha),
$$

and it can be simplified approximating the cosine function with its Taylor expansion up to the second term, such that $\cos (\alpha) \approx 1-\alpha^{2} / 2$. After few simple algebraic operations, the now approximated $D^{2}$ can be expressed as follows:

$$
D^{2}=(|y|-|x|)^{2}+|x| \cdot|y| \cdot \alpha^{2} .
$$




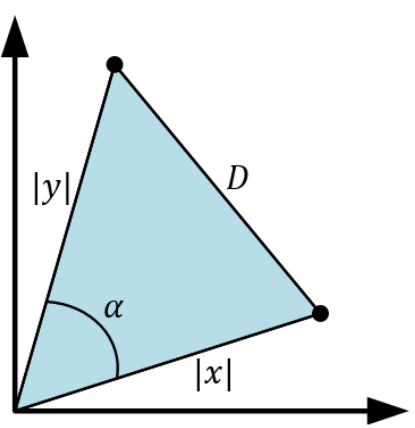

(a)

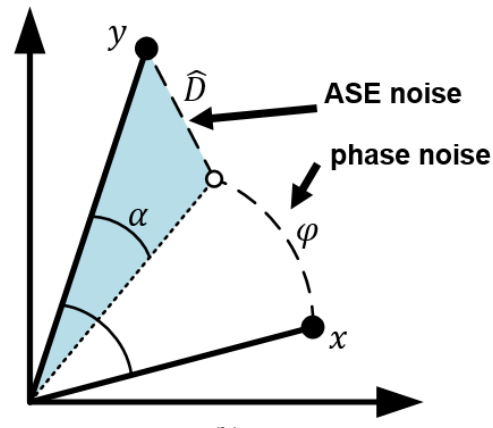

(b)

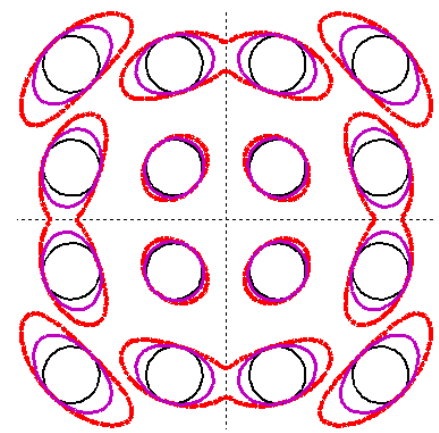

(c)

Fig 1. (a) Triangle formed by 2 points and the origin. (b) Composition of ASE and phase noise (c) Example set of equidistant curves in a 16QAM

Replacing Eq. (4) into Eq. (3) and assuming the sample-by-sample approach (i.e. $\varphi(k-1)=0$ ), the log-probability function to be minimized becomes as follows

$$
\log (P)=\frac{(|y|-|x|)^{2}+|x| \cdot|y| \cdot \alpha^{2}}{2 \sigma_{n}{ }^{2}}+\frac{(\arg (y)-\arg (x)-\alpha)^{2}}{2 \sigma_{p}^{2}} .
$$

Note that in Eq. (5) the phase noise component is implicitly denoted as $\varphi=\arg (y)-\arg (x)-\alpha$ (see Fig. 1(b)). After differentiating Eq. (5) w.r.t. $\alpha$ and equaling to zero, the angle $\alpha$ that minimizes Eq. (5) is found to be

$$
\alpha=\frac{\arg (y)-\arg (x)}{k|x||y|+1},
$$

where $k=\sigma_{p}^{2} / \sigma_{n}^{2}$. Finally, replacing the solved angle $\alpha$ in Eq. (4), it is possible to write the proposed pMAP distance (squared) as follows:

$$
D^{2}(x, y, k)=(|y|-|x|)^{2}+\left(\frac{|x||y|}{(k|x||y|+1)^{2}}\right)(\arg (y)-\arg (x))^{2} .
$$

Fig. 1(c) shows an example of equidistant curves for three values of $k$ using Eq. (7) for a 16QAM constellation.

\section{Simulation Investigations}

\section{Simulation setup}

To verify the effectiveness of the new metric in CPE, the performance of BPS algorithm is evaluated with the proposed pMAP metric and compared against the conventional Euclidean distance through extensive simulations. The tested modulation formats are 4-, 16-, 64-, and 256-QAM with differential encoding/decoding as described in [4]. Data is generated according to Eq. (1) for different values of signal-to-noise ratio (SNR) and $\Delta v T_{s}$, and fed into BPS unit which is implemented as in [4] where for each symbol the decision $\hat{x}(k)$ is made by finding the minimum sum:

$$
s(k, b)=\sum_{n=-N}^{N} D^{2}(k-n, b),
$$

where $D$ is either Euclidean or pMAP distance as in Eq. (7), $2 N+1$ is the filter length and $b \in\{0,1 . \cdots, B-1\}, B$ being the number of test angles. In this work, we use $2 \times 10^{5}$ symbols for all data points, $N=5$, and $B=32$.

\section{Results and discussion}

To evaluate the performance, SNR penalty from theoretically achievable sensitivity with differential coding is calculated. Fig. 2 shows the measured SNR penalty at bit error ratio (BER) of $10^{-3}$ and it can be observed, except for QPSK - where due to symmetry both metrics make no difference- the linewidth tolerance limits exceed those achieved when using Euclidean distance. The linewidth enhancement achieved by using the proposed pMAP metric increases also for higher SNR penalties.

Also the measured SNR penalty at a lower BER of $10^{-2}$ is shown in Fig. 3. Again, with the exception of QPSK, the pMAP metric is shown to improve the achievable linewidth tolerance for higher-order QAM constellations.

Table I summarizes the achievable limits for $\Delta v T_{s}$ and the equivalent maximum combined laser linewidth figures corresponding to a system operating at 32 Gbaud, for $1 \mathrm{~dB}$ SNR penalty at BER values of $10^{-3}$ and $10^{-2}$. 


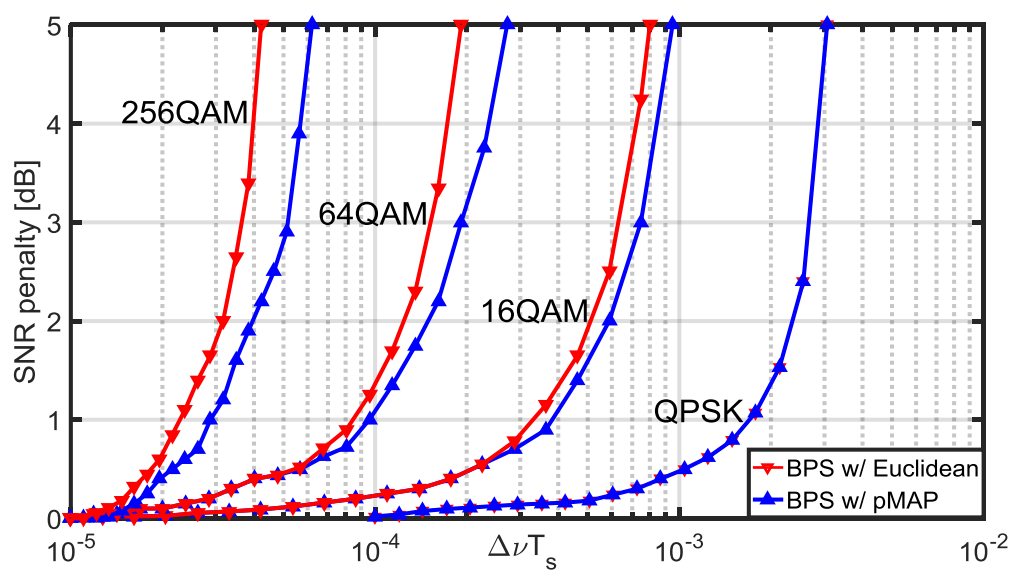

Fig 2. SNR penalty for QPSK, 16QAM, 64QAM, 256QAM at a BER of $10^{-3}$

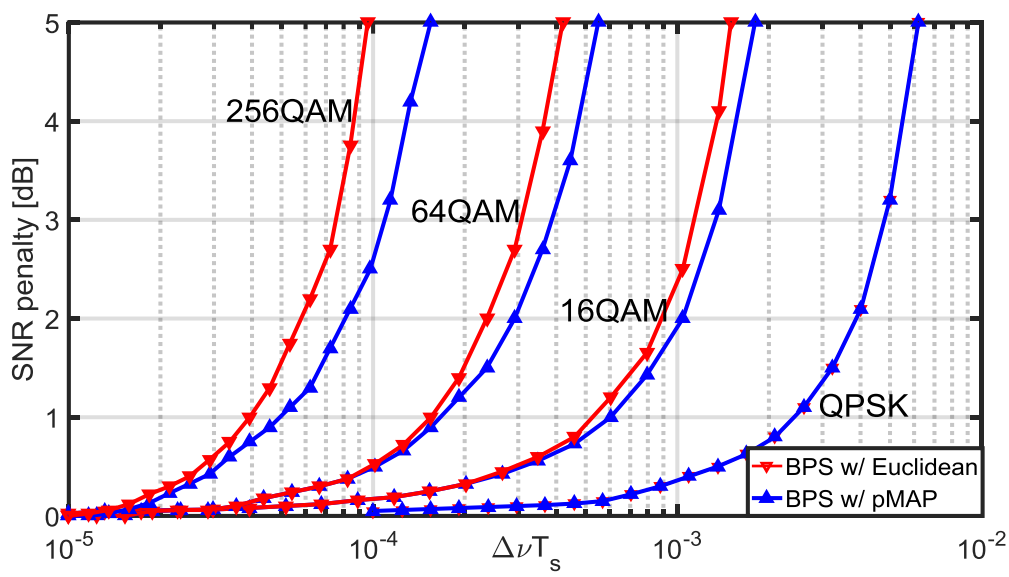

Fig 3. SNR penalty for QPSK, 16QAM, 64QAM, 256QAM at a BER of $10^{-2}$

TABLE I. SUMMARY OF MAXIMUM TOLERABLE LINEWIDTHS

\begin{tabular}{|c|c|c|c|c|c|c|c|c|}
\hline \multirow{2}{*}{$\begin{array}{c}\text { Modulation } \\
\text { format }\end{array}$} & \multicolumn{3}{|c|}{ 1dB SNR penalty @ BER $=10^{-3}$} & \multicolumn{3}{c|}{ 1dB SNR penalty @ BER $=10^{-2}$} \\
\cline { 2 - 8 } & \multicolumn{2}{|c|}{ max tolerable $\Delta v T_{s}$} & \multicolumn{2}{c|}{ max $\Delta v @ 32 \mathrm{GBaud}$} & \multicolumn{2}{c|}{ max tolerable $\Delta v T_{s}$} & \multicolumn{2}{c|}{ max $\Delta v @ 32 \mathrm{GBaud}$} \\
\hline & Euclidean & $\mathrm{pMAP}$ & Euclidean & pMAP & Euclidean & pMAP & Euclidean & pMAP \\
\hline 4-QAM & \multicolumn{2}{|c|}{$1.7 \times 10^{-3}$} & \multicolumn{2}{|c|}{$54.4 \mathrm{MHz}$} & \multicolumn{2}{|c|}{$2.5 \times 10^{-4}$} & \multicolumn{2}{c|}{$8 \mathrm{MHz}$} \\
\hline 16-QAM & $3.3 \times 10^{-4}$ & $3.8 \times 10^{-4}$ & $10.6 \mathrm{MHz}$ & $12.2 \mathrm{MHz}$ & $5 \times 10^{-4}$ & $6 \times 10^{-4}$ & $16 \mathrm{MHz}$ & $19.2 \mathrm{MHz}$ \\
\hline 64-QAM & $0.8 \times 10^{-4}$ & $1 \times 10^{-4}$ & $2.56 \mathrm{MHz}$ & $3.2 \mathrm{MHz}$ & $1.5 \times 10^{-4}$ & $1.7 \times 10^{-4}$ & $4.8 \mathrm{MHz}$ & $5.44 \mathrm{MHz}$ \\
\hline 256-QAM & $2.3 \times 10^{-5}$ & $3 \times 10^{-5}$ & $736 \mathrm{kHz}$ & $960 \mathrm{kHz}$ & $3.9 \times 10^{-5}$ & $5 \times 10^{-5}$ & $1.25 \mathrm{MHz}$ & $1.6 \mathrm{MHz}$ \\
\hline
\end{tabular}

\section{Conclusions}

A new polar-based metric to analytically solve the sample-by-sample MAP estimate in presence of both ASE and phase noise has been presented. Through simulations it has been shown that replacing the Euclidean distance with the presented pMAP metric in the BPS algorithm, it is possible to enhance the linewidth tolerance for high-order QAM modulation formats.

\section{Acknowledgment}

This work was supported by EU project ICONE, grant number 608099.

\section{References}

[1] D. Lavery, R. Maher, D. S. Millar, B. C. Thomsen, P. Bayvel and S. J. Savory, "Digital coherent receivers for long-reach optical access networks," J. Lightwave Technol., 31(4), 609-620 (2013).

[2] A. Viterbi, "Nonlinear estimation of PSK-modulated carrier phase with application to burst digital transmission," IEEE Trans. Inf. Theory, 29(4), 543-551 (1983)

[3] M. G. Taylor, "Phase estimation methods for optical coherent detection using digital signal processing," J. Lightwave Technol., 27(7), 901914 (2009).

[4] T. Pfau, S. Hoffmann and R. Noe, "Hardware-efficient coherent digital receiver concept with feedforward carrier recovery for $M$-QAM constellations," J. Lightwave Technol., 27(8), 989-999 (2009). 